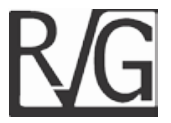

\title{
Estructura económico-financiera de pequeñas y medianas empresas manufactureras colombo-venezolanas
}

\author{
Oliveros Delgado, Juan Alberto*
}

\section{Resumen}

Con el apoyo de herramientas de análisis de estados financieros, el presente artículo busca contrastar los rasgos de la estructura económica y financiera de las Pequeñas y Medianas Empresas Manufactureras de la zona fronteriza colombo-venezolana, más específicamente de Cúcuta y San Cristóbal, respectivamente, durante los años 2009 al 2012. La investigación es descriptiva, documental, con muestreo intencional. Entre los principales hallazgos destaca que las empresas venezolanas estudiadas concentran su estructura económica en partidas no monetarias, aplican políticas conservadores de crédito y se financian principalmente con la banca ante tasas de interés reales negativas. En contraparte, las empresas colombianas mantienen una proporción mucho mayor en cuentas monetarias, flexibilizan sus políticas de crédito y se financian mayormente por la vía comercial. Se concluye que el escenario macroeconómico impacta en la estructura económicofinanciera de las organizaciones; estas deben ajustarse buscando mantener su efectividad. Aun cuando el parque industrial de San Cristóbal genera comparativamente mejores resultados, en términos de rentabilidad y beneficio, estos se inciden negativamente ante un ambiente de creciente inflación.

Palabras clave: estructura económico-financiera; análisis financiero; pequeña y mediana industria.

Recibido: 25-02-14 Aceptado: 20-6-15

* Ingeniero Industrial, Msc en Gerencia Financiera, Profesor Asistente, Universidad Nacional Experimental del Táchira/Universidad Católica del Táchira. Investigador acreditado por el Programa de Estímulo al Investigador e Innovador (PEII), 2013. E-mail: jaoliveros@unet.edu.ve 


\title{
Economic-financial structure of small and medium sized Colombian-Venezuelan manufacturing companies
}

\begin{abstract}
With the support of financial statements analysis tools, the present research is aimed to contrast the economic and financial structure features of Small and Medium Sized Manufacturing Companies from the Colombian-Venezuelan border, more specifically from Cúcuta and San Cristobal respectively from 2009 to 2012, The research is descriptive, documental, with intentional sampling. Among the main findings is highlighted that the studied Venezuelan companies concentrate their economic structure in non monetary items, apply conservative credit policies and are principally financed with the banking at negative real interest rates. In counterpart, Colombian companies maintain a much higher proportion on monetary accounts, stretch their credit policies and are mostly financed by the commercial route. It is concluded that the macroeconomic scenery impacts on the economic-financial structure of the organizations; this should be adjusted seeking to maintain their effectiveness. Even when the industrial park of San Cristobal comparatively generates better results, this are negatively influenced in terms of profitability and benefit in front of an increasing inflation environment.
\end{abstract}

Key Words: economic-financial structure; financial analysis; small and medium sized industries.

\section{Introducción}

El estudio de las Pequeñas y Medianas Empresas (PYMES) es un tema de investigación inagotable y objeto de publicaciones frecuentes (Vera-Corina, 2008). Dichas investigaciones pretenden la mejora continua de estas organizaciones en sus distintos ámbitos, la identificación de sus características, análisis de sus problemáticas y la profundización de su realidad actual. La importancia de este tipo de empresas queda en evidencia a través de variables como contribución al Producto Interno Bruto (PIB), generación de empleo y valor agregado, fomento del empleo formal, movilidad entre los estratos sociales, calificación de la mano de obra y mejor distribución del ingreso.
Como agente generador de valor agregado, es la PYME Manufacturera, denominada Pequeña y Mediana Industria (PYMI) la que por su actividad transformadora de materias primas juega un papel vital como elemento dinamizador de la economía y la sociedad.

En Venezuela, la definición de PYMI, quedó legalmente establecida en el artículo quinto de la Ley para la Promoción y Desarrollo de la Pequeña y Mediana Industria y demás Unidades de Producción Social (Presidencia de la República, 2008), como aquellas unidades productivas que ejercen actividades de transformación de insumos, con una nómina promedio anual no mayor de 100 trabajadores y una facturación anual de hasta 250.000 Unidades Tributarias (UT) 1 .

1 La unidad tributaria es una medida de valor creada a efectos fiscales, equivalente a cierto monto en unidades monetarias, y sobre la base del Índice de Precios al Consumidor (Presidencia de la República, 1994). 
En Colombia, la Pequeña y Mediana Industria no encuentra una definición formal, el término queda implícito bajo la denominación Pequeña y Mediana Empresa del sector Manufacturero. Una definición aún vigente, es ofrecida a través de la Ley 905 (Congreso de la República de Colombia, 2004) sobre promoción del desarrollo de la Micro, Pequeña y Mediana Empresa Colombiana, que en su artículo número 02 , las cataloga como entidades de producción manufacturera con una planta de personal inferior a doscientos trabajadores $\mathrm{o}$ activos totales de hasta treinta mil salarios mínimos vigentes.

Las definiciones anteriores enmarcan los dos grupos de producción considerados en el presente estudio, ambos, son objeto de políticas públicas de fomento, en cada uno de sus países, sin embargo deben enfrentar una serie creciente de obstáculos que amenazan su operatividad y permanencia en el mercado. En el ámbito venezolano, una lista no exhaustiva de dificultades y amenazas fue presentada por el Presidente de la Cámara de la Industria de la Transformación de México, Cuauhtémoc Martínez, en el marco del XXXVI Encuentro Internacional de la Confederación Venezolana de Industriales (CONINDUSTRIA), celebrado en el año 2006:

"Baja productividad y falta de competitividad, reducidos niveles de capitalización, planificación financiera improvisada, inadecuada administración financiera, escaso o nulo acceso a esquemas de crédito e incentivos, falta de capacitación laboral y gerencial, uso de tecnologías obsoletas, inadecuada organización interna, ausencia de redes y asociación entre las empresas, dependencia externa de insumos, maquinaria y equipos, irregularidad administrativa y normativa".

Se suma a lo expuesto, según el Presidente de la Asociación de Micros,
Pequeños y Medianos empresarios de Venezuela (Gómez, Entrevista 2014), la creciente escasez de insumos y la dificultad cada vez mayor para acceder a las divisas que permita la adquisición de materias primas y tecnologías provenientes del extranjero, con el consecuente impacto en los costos. El caso Colombiano, no es ajeno a las debilidades y algunas de las amenazas antes expuestas. Montoya et al (2010:4) enuncian una serie de factores que han limitado la competitividad de las PYME Colombianas: "Poca sofisticación y baja agregación de valor en sus proceso productivos, baja productividad y capacidad de generación de empleo formal, alta informalidad, atraso del mercado financiero, deficiente infraestructura de transportación y energía, y un sistema tributario poco amigable". La problemática descrita sobre las empresas de ambos países representa un alegato suficiente para proponer e implementar políticas y estrategias concretas en pro de la sostenibilidad de tan importantes entidades.

En cuanto a sustentabilidad, un estudio auspiciado por el Banco Interamericano de Desarrollo (BID) a través del Observatorio de Micro Pequeña y Mediana Empresa reporta que un tercio de los registros efectuados por pequeños y medianos empresarios en América Latina, cierran sus puertas o se declaran insolventes durante sus dos primeros años de actividad (BID, 2007). De los establecimientos que cierran sus puertas según Stangl (2011:03) cerca de un 50\% lo hace por problemas relacionados con las ventas, un $45 \%$ por situaciones financieras y un $5 \%$ por causas administrativas, estas últimas cuando no son solucionadas oportunamente se convierten en complicaciones financieras difíciles de resolver. 
De acuerdo a lo expresado en párrafos anteriores, el presente artículo representa una iniciativa empírica para contrastar los rasgos de la estructura económica y financiera de las pequeñas y medianas empresas manufactureras, específicamente los pequeños y medianos parques industriales de San Cristóbal (Venezuela) y Cúcuta (Colombia); a través de un análisis financiero tradicional que describe y exalta su estructuración de activos, estructura financiera y los módulos de solvencia, actividad, endeudamiento y rentabilidad.

Adicionalmente, se muestra cómo elementos del entorno macroeconómico ejercen influencia sobre la estructuración económico-financiera de las PYMI y sus resultados.

La investigación realizada presenta un carácter cuantitativo y diseño documental, toma como principal insumo la información contable proveniente de los estados financieros; adicionalmente un nivel descriptivo, a través de la caracterización los principales rasgos financieros de las PYMI objeto de estudio. Se procuró hacer semblanza de cómo cada grupo conforma su estructura de activos, pasivos, patrimonio, niveles de solvencia, endeudamiento, administración del capital de trabajo y finalmente la rentabilidad.

\section{Algunas consideraciones teóricas sobre análisis financiero}

El análisis financiero, según Ortega (2002:397) es una rama del saber financiero, cuyos fundamentos y objetivos giran en torno a la obtención de medidas y relaciones cuantitativas para la toma de decisiones, a través de la aplicación de instrumentos y técnicas matemáticas sobre cifras y datos suministrados por la contabilidad, transformándolos para su debida interpretación.

Rivera y Ruíz (2011), amplían las fuentes y maneras de efectuar un diagnóstico financiero argumentando la existencia de al menos tres metodologías para conocer el desempeño de las organizaciones basadas en: a) información contable, b) información del mercado bursátil, y c) criterios de la gerencia del valor. La más tradicional y comúnmente usada es la contable; la de mercado es de acceso casi exclusivo para las empresas que cotizan en bolsa y economías de alta bursatilidad. Por su parte, la metodología fundamentada en el uso de la gerencia del valor, aunque ha venido en aumento en los últimos años, no es tan popularizada.

La información contable como fuente universal para el análisis proporciona una resumida y elaborada información para la toma de decisiones, García (2001:184) sintetiza con claridad que el análisis de estados financieros implica efectuar un importante número de operaciones matemáticas para:

- Obtener porcentajes de cada partida contable respecto a su rubro integral (análisis vertical) estimando así cuáles son las partidas de mayor peso o participación tanto en el estado de situación financiera como en estado de resultados. Esto es estimar las partidas más representativas.

- Calcular las variaciones de los saldos de las partidas más representativas a través de los años y determinar los porcentajes de cambio (análisis horizontal) buscando conocer cómo tales variaciones inciden en la gestión económica financiera de la entidad.

- Finalmente, relacionar partidas o grupos de partidas del estado de situación financiera y/o del estado de 
resultados a través de operaciones aritméticas determinando las llamadas razones o indicadores.

Si García (2001) define una razón financiera como la relación entre dos o más cuentas contables del estado de situación financiera o del estado de resultados, entonces habrá tantas razones como combinación de cuentas exista. Para que su uso sea efectivo, deberá limitarse su número en función al interés del usuario y los objetivos del análisis.

Gitman y Zutter (2012:65) destaca la conveniencia, de agrupar las razones atendiendo al objetivo del análisis, en categorías básicas: liquidez, actividad, endeudamiento y rentabilidad; las tres primeras miden riesgo del negocio, la última mide el rendimiento. Seguidamente se describen cada una de ellas.

Módulo de solvencia: Según Ortega (2002:03) el estudio de la solvencia mide la capacidad del negocio para cubrir sus compromisos a corto plazo. Lo cual, puede ser evaluado en base a tres razones básicas cuya fórmula e interpretación se resume en el Cuadro 1.

\section{Cuadro 1}

Razones básicas para evaluar la solvencia

\begin{tabular}{ccl}
\hline Indicador & \multicolumn{1}{c}{ Fórmula } & \multicolumn{1}{c}{ Interpretación } \\
\hline $\begin{array}{c}\text { Solvencia } \\
\text { Corriente }\end{array}$ & $\begin{array}{l}\text { Activo circulante } \\
\text { Pasivo circulante }\end{array}$ & $\begin{array}{l}\text { Capacidad de la empresa para cubrir sus pasivos } \\
\text { a corto plazo a partir de su activo disponible, } \\
\text { exigible y realizable. }\end{array}$ \\
Liquidez & $\begin{array}{lll}\text { Activo circulante } \text {-inventarios } \\
\text { Pasivo circulante }\end{array}$ & $\begin{array}{l}\text { Capacidad de la empresa para hacer frente a } \\
\text { sus compromisos a corto plazo sin hacer uso del } \\
\text { inventario (recurriendo sólo al activo disponible y } \\
\text { exigible) }\end{array}$ \\
Solidez & Activos totales & $\begin{array}{l}\text { Posición de la empresa para hacer frente a la } \\
\text { totalidad de sus compromisos haciendo uso de la } \\
\text { totalidad de sus activos. }\end{array}$
\end{tabular}

Fuente: Elaboración propia, a partir de Ortega (2002)

Módulo de endeudamiento: los indicadores de este módulo permiten medir el riesgo financiero que soporta la empresa, esto es, cuán endeudada se encuentra respecto al patrimonio de los accionistas. Su importancia según Lizcano (2004:54) radica en el hecho de que el riesgo de insolvencia aumenta en la medida en que se incrementan las deudas o recursos ajenos de la empresa respecto al capital propio o los recursos internos. Dicho nivel de riesgo puede ser medido a través de las razones presentadas en el Cuadro 2. 


\section{Cuadro 2}

\section{Razones financieras para evaluar el nivel de endeudamiento}

\begin{tabular}{|c|c|c|}
\hline Indicador & Fórmula & Interpretación \\
\hline & & $\begin{array}{l}\text { Indicador del riesgo financiero de la empresa; relaciona } \\
\text { el total de los recursos ajenos respecto a los recursos } \\
\text { propios. Comúnmente se definen cuatro categorías: }\end{array}$ \\
\hline Endeudamiento & Pasivo Total /Patrimonio & $\begin{array}{ll}\text { - } & \text { Sub-endeudamiento }(\leq 50 \%) \\
\text { - } & \text { Normal }(>50 \% \leq 100 \%) \\
\text { - } & \text { Moderado }(>100 \% \leq 200 \%) \\
\text { - } & \text { Sobreendeudamiento }(>200 \%)\end{array}$ \\
\hline $\begin{array}{l}\text { Apalancamiento } \\
\text { (D) }\end{array}$ & Pasivo /Activo & \multirow{2}{*}{$\begin{array}{l}\text { Ambos indicadores son complementarios y caracterizan } \\
\text { la estructura financiera de la empresa; representan la } \\
\text { proporción de los activos que está siendo financiada por } \\
\text { capital externo e interno respectivamente. }\end{array}$} \\
\hline $\begin{array}{l}\text { Razón Autonomía } \\
\qquad(\mathrm{E})\end{array}$ & Patrimonio/Activo & \\
\hline
\end{tabular}

Fuente: Elaboración propia.

Módulo de actividad: Según Gitman y Zutter (2012:68), estos indicadores miden la rapidez con que las cuentas corrientes de mayor relevancia se convierten en ventas o efectivo. Se asocian a la eficiencia con la que opera una empresa en una variedad de dimensiones, como la administración de inventarios, gastos y cobros, permitiendo conocer cuán eficiente es la gestión del capital de trabajo. Un resumen de dichas razones se detalla en el Cuadro 3.

\section{Cuadro 3}

\section{Razones para evaluar los niveles de actividad}

\begin{tabular}{|c|c|c|}
\hline Indicador & Fórmula & Interpretación \\
\hline $\begin{array}{l}\text { Días medios de cobro } \\
\qquad(D A M C X C) \\
\text { Días medios de } \\
\text { inventario }(D A M \text { Inv) }\end{array}$ & $\begin{array}{c}\frac{\text { Ctas. por cobrar comerciales }}{\text { Ingreso neto }} \times 360 \\
\frac{\text { Inventario neto }}{\text { Costo de ventas }} \times 360\end{array}$ & $\begin{array}{l}\text { Están asociadas al grado de liquidez o } \\
\text { convertibilidad en efectivo de las partidas } \\
\text { de activos circulantes. Determinan qué tan } \\
\text { realizables son los inventarios y qué tan } \\
\text { exigibles las cuentas por cobrar. }\end{array}$ \\
\hline $\begin{array}{l}\text { Días medios de } \\
\text { cuentas por pagar } \\
(D A M C X P)\end{array}$ & $\frac{\text { Ctas. por cobrar proveedores }}{\text { Costo de ventas }} \times 360$ & $\begin{array}{l}\text { Determina qué tan frecuentemente la empresa } \\
\text { honra compromisos con proveedores. }\end{array}$ \\
\hline Ciclo operativo & $D A M C \times C+D A M$ Inventario & $\begin{array}{l}\text { Permite conocer el plazo promedio en días que } \\
\text { demora el efectivo en retornar a caja pasando } \\
\text { por todas las etapas propias del giro normal del } \\
\text { negocio. (Compras, procesamiento, almacén, } \\
\text { facturación, despacho, cobranza.) }\end{array}$ \\
\hline Ciclo de la Caja & Ciclo Operativo - DAM CXP & $\begin{array}{l}\text { Estimador de las necesidades de previsión de } \\
\text { efectivo para fines operacionales o del plazo en } \\
\text { días que se requerirá financiar capital de trabajo. }\end{array}$ \\
\hline
\end{tabular}

Fuente: Elaboración propia, a partir de Ortega (2002) 
Módulo de rentabilidad: Ortega (2002:11) define rentabilidad como: "la capacidad que posee un negocio para generar utilidades en función a los recursos empleados, económicos o financieros". Permite medir la eficiencia de la inversión de los accionistas, la eficiencia de los activos empleados y en una forma sofisticada si los resultados del negocio superan al costo promedio de los capitales empleados. Una forma de cálculo e interpretación de las razones que agrupa este módulo se muestra en el Cuadro 4.

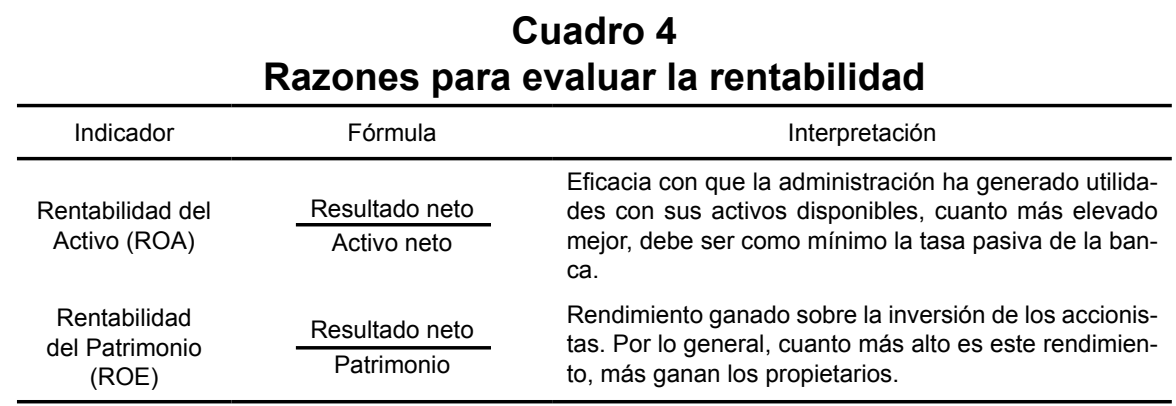

Fuente: Elaboración propia.

Finalmente, vale la pena discernir entre estructura de activos y estructura financiera. La primera está íntimamente relacionada con las decisiones de inversión, o el conjunto de inversiones realizadas por las empresas. La segunda (estructura financiera) según Sánchez (2004:2), corresponde a la forma cómo la empresa financia sus inversiones, incluye la deuda tanto de corto como de largo plazo (pasivos) así como el patrimonio de los accionistas.

\section{Estructura económico- financiera de las PYMI colombo- venezolanas: características más relevantes}

Antes de caracterizar la estructura económico-financiera de las PYMI estudiadas, se describe la metodología empleada para la determinación de las empresas que conforman la muestra objeto de estudio y la recolección de la data financiera necesaria.
En el municipio de San Cristóbal, no se dispone de directorios que mantengan información actualizada acerca de la población o universo de estudio, la data institucional más reciente ubicada hasta la fecha corresponde al IV Censo Económico Empresarial desarrollado por el Instituto Nacional de Estadística (INE) entre los años 2007 y 2008, publicado de manera parcial en el año 2010 (INE, 2010), fuente según la cual en el municipio operaban 149 pequeñas y medianas industrias para el momento del estudio.

En el caso de las PYMI ubicadas en Cúcuta, se cuenta con varias fuentes de información, entre ellas el Departamento Administrativo Nacional de Estadística (DANE) y la base de datos del Sistema de Información y Reporte Empresarial (SIREM) creado y actualizado por la Superintendencia de Sociedades. Por iniciativa privada, existen directorios empresariales como el ofrecido por Informa Colombia S.A.; según este último, en el Departamento Norte de Santander se cuentan a 
mayo del 2013 con 459 establecimientos manufactureros registrados, desde micro hasta grandes industrias; de los cuales, 146 corresponden a las PYMI que ejercen su actividad en la capital del Departamento, Cúcuta.

Gracias al SIREM (Superintendencia de Sociedades, 2013) es posible acceder de forma instantánea, vía web a la información financiera de las empresas que reportan operatividad a la Superintendencia de Sociedades, data disponible en formato de Microsoft Excel. La información financiera de las PYMl cucuteñas comprende los años 2009 al 2012.

En el caso del municipio San Cristóbal, no se cuenta con tal grado accesibilidad. La información contable de las PYMI es confidencial, por lo que la data fue recabada de forma progresiva, durante los años antes mencionados, con apoyo de estudiantes de pregrado de la Universidad Católica del Táchira y la Universidad Nacional Experimental del Táchira, contando con el consenso de los representantes legales o administradores de las empresas, manteniendo la denominación y razón social en anonimato.

Tanto el tamaño de la muestra, como la selección de los sujetos muestrales (conformación de la muestra) mantuvieron un carácter intencional, procurando proporcionalidad en cuanto a la presencia de distintos subsectores industriales de acuerdo a la Codificación Internacional Industrial Uniforme: alimentos y bebidas, textil y calzado, plástico y fibras, metalmecánico, químicos, papel, edición e impresión. De esta forma se conformaron muestras discrecionales de 112 empresas del lado $\mathrm{Cu}$ cuteño y 76 para San Cristóbal.

La recolección de la información financiera se llevó a cabo mediante una ficha elaborada en Microsoft Excel, la cual recoge las cuentas contables suficientes para cubrir los objetivos planteados. Para el procesamiento de los datos se tabuló la información financiera según la ficha resumen y se totalizaron cada una de las cuentas, algunas de las cuales por su poca representatividad fueron reclasificadas en "otros" (cargos diferidos y depósitos en garantía entre los activos; pasivos contingentes y diferidos en los pasivos) generando luego estados financieros porcentuales, bajo el procedimiento conocido en contabilidad gerencial como análisis vertical.

Definidos los aspectos metodológicos se presentan paulatinamente los resultados. En primer lugar se reseña la estructura de activos de las PYMI, íntimamente relacionada con las decisiones de inversión, lo cual, se detalla en términos porcentuales en la Tabla 1.

\section{Tabla 1}

\section{Estructura de inversión de las PYMI colombo-venezolanas (Período 2009-2012)}

\begin{tabular}{ccccccccccc}
\hline & \multicolumn{4}{c}{$\begin{array}{c}\text { Análisis vertical de las } \\
\text { PYMIde San Cristóbal (\%) }\end{array}$} & \multicolumn{5}{c}{$\begin{array}{c}\text { Análisis vertical de las } \\
\text { PYMI de Cúcuta (\%) }\end{array}$} \\
\hline ACTIVO & $\mathbf{2 0 0 9}$ & $\mathbf{2 0 1 0}$ & $\mathbf{2 0 1 1}$ & $\mathbf{2 0 1 2}$ & Prom & $\mathbf{2 0 0 9}$ & $\mathbf{2 0 1 0}$ & $\mathbf{2 0 1 1}$ & $\mathbf{2 0 1 2}$ & Prom \\
\hline Activo corriente & 59,85 & 48,65 & 54,04 & 41,01 & $\mathbf{5 0 , 8 9}$ & 59,61 & 58,01 & 60,33 & 65,74 & $\mathbf{6 0 , 9 2}$ \\
Activo fijo (Neto) & 30,58 & 43,01 & 34,80 & 34,24 & $\mathbf{3 5 , 6 6}$ & 30,46 & 27,11 & 23,43 & 21,64 & $\mathbf{2 5 , 6 6}$ \\
Otros activos & 9,57 & 8,35 & 11,15 & 24,75 & $\mathbf{1 3 , 4 5}$ & 9,94 & 14,88 & 16,24 & 12,62 & $\mathbf{1 3 , 4 2}$ \\
\hline
\end{tabular}

Fuente: elaboración propia, a partir de información financiera de datos del SIREM (Colombia) y obtenidos de la aplicación de encuestas a empresas venezolanas. 
La traducción a términos porcentuales de las principales cuentas del activo respecto al total devela como las PYMI colombianas durante el período 2009-2012 destinan en promedio una mayor proporción de fondos a los activos corrientes 0 circulantes $(60,92 \%)$, traducidos estos últimos en sólo tres cuentas: el activo disponible (efectivo y equivalentes) exigible (cuentas por cobrar comerciales) y realizable (inventarios), más adelante se ofrece el detalle de estas partidas; obsérvese como el promedio para los cuatro años, en el caso colombiano, supera en más de diez puntos porcentuales al venezolano.

Las cifras también permiten apreciar que las empresas venezolanas durante el período 2009-2012 mantienen una mayor inversión de fondos en activos fijos, con un promedio de un $35,66 \%$ con tendencia estable. En el caso colombiano, el referido valor en promedio es diez puntos porcentuales inferior $(25,66 \%)$, observándose para los cuatro años una marcada tendencia decreciente, lo descrito puede estar vinculado a la naturaleza no monetaria de la partida de activos fijos y la práctica de proteger la estructura económica ante ambientes inflacionarios (caso venezolano).

El detalle del activo corriente del promedio del período 2009-2012 se muestra y se compara a través de la Figura 1 , en primer lugar se evidencia como el parque industrial de San Cristóbal mantiene en términos porcentuales mayores niveles de efec-tivo en comparación a sus homólogas cucuteñas. Según Higuerey (2007:61) existen tres necesidades para mantener efectivo, un nivel transaccional correspondiente al efectivo para atender las operaciones diarias (nómina, compras, pago de cuentas, impuestos) un nivel de precaución, para afrontar imprevistos ocasionales y un nivel de especulación el cual, busca aprovechar descuentos y economía de escala.

\section{Figura 1}

\section{Conformación de los activos corrientes de las PYMI colombo-venezolanas (Promedio del período 2009-2012)}
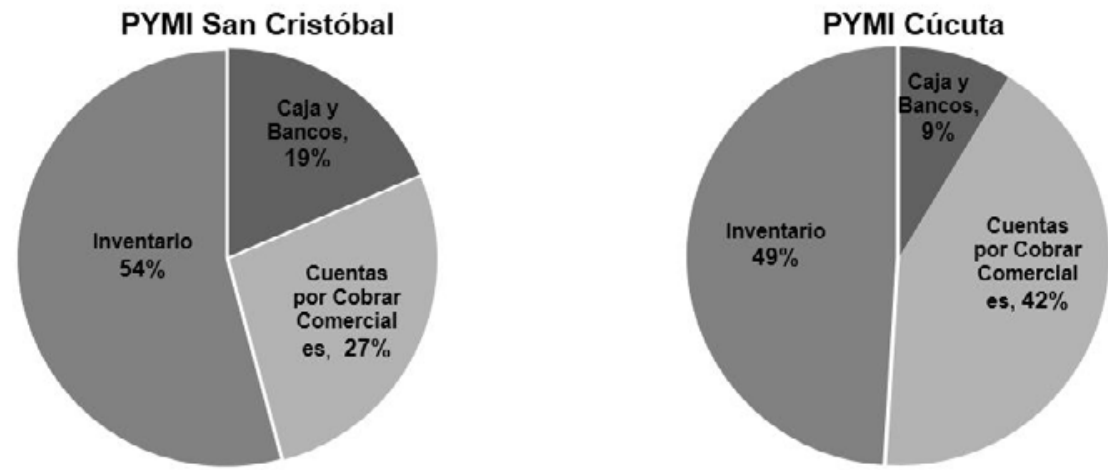

Fuente: elaboración propia, a partir de información financiera obtenida del SIREM (Colombia) y de la aplicación de encuestas a empresas venezolanas. 
En las empresas venezolanas puede atribuirse esta diferencia en cuanto al nivel de efectivo a motivos especulativos y de precaución. Mayor diferenciación se muestra en el porcentaje de las cuentas por cobrar, donde el parque venezolano resulta mucho más conservador, mientras sólo un $14 \%$ de los activos son cuentas por cobrar para las PYMI de San Cristóbal, en las industrias de Cúcuta este porcentaje ronda un $26 \%$ y representa la segunda partida de mayor participación en estado de situación financiera luego de los inventarios. Posteriormente se expondrá cómo esta configuración repercute en los índices de actividad y ciclo de caja.

Otra característica importante de destacar es la jerarquía de las cuentas de activos dentro de la estructura económica, esto es, para las PYMI de San Cristóbal la principal partida dentro del estado de situación financiera son los activos fijos, netos de depreciación. A valores nominales estos representan cerca de un $36 \%$ del total activo, propio de empresas industriales; le sigue la partida de inventarios con un $27,6 \%$, ambas concentran un $63,6 \%$ de la inversión en activos; como partidas no monetarias mantienen su valor ante ambientes inflacionarios y más aún se revaloran.

En el caso de las PYMI de Cúcuta la primera partida es el inventario con un $29,8 \%$ del total activo, le siguen las cuentas por cobrar comerciales con un $25,8 \%$ y en tercer lugar aparece el activo fijo neto con un $25,6 \%$ del total activo. Sorprende como una cuenta monetaria como es el caso de las cuentas por cobrar supere incluso la inversión en activos fijos operacionales. Al respecto cabe mencionar que en un mercado de libre competencia, una estrategia para preservar e incluso impulsar las ventas, es el crédito, aun cuando trae implícito costos y riesgos.

Tal conformación en cuanto a decisiones de inversión, puede hallar explicación en el efecto que impone el entorno Macroeconómico, comparativamente para ambos países refleja marcadas diferencias, solo respecto a la inflación Venezuela ha preservado una cifra atípica a la del resto de países de América con un promedio de un 25\% anual hasta el 2012 (Banco Central de Venezuela, 2012), entretanto Colombia ostenta una reducción progresiva del índice general de precios cerrando el 2012 con apenas un 2,44\% anual (DANE, 2013). El comportamiento de la variable puede explicar en buena parte la conformación de la estructura de activos para las empresas analizadas, ya que como se mencionó anteriormente la práctica comúnmente aceptada en finanzas organizacionales ante economías altamente inflacionarias es resguardar la posición económica vía partidas no monetarias.

En cuanto a la estructura financiera, representada por la deuda, tanto de corto como de largo plazo, así como el patrimonio de los accionistas, se expone en las Tablas 2 y 3 . La primera incluye lo concerniente a los pasivos tanto corrientes como no corrientes expresados en términos porcentuales respecto al pasivo total. 


\section{Tabla 2}

\section{Estructura de las fuentes de financiamiento externas de las PYMI colombo-venezolanas (Período 2009-2012)}

\begin{tabular}{|c|c|c|c|c|c|c|c|c|c|c|}
\hline \multirow[b]{2}{*}{ PASIVO } & \multicolumn{5}{|c|}{$\begin{array}{c}\text { Estructura del pasivo de las PYMI } \\
\text { de San Cristóbal (\%) }\end{array}$} & \multicolumn{5}{|c|}{$\begin{array}{c}\text { Estructura del pasivo de las PYMI } \\
\text { de Cúcuta (\%) }\end{array}$} \\
\hline & 2009 & 2010 & 2011 & 2012 & Prom & 2009 & 2010 & 2011 & 2012 & Prom \\
\hline $\begin{array}{l}\text { Cuentas } \\
\text { por pagar } \\
\text { comerciales }\end{array}$ & 29,11 & 22,23 & 27,60 & 20,87 & 24,95 & 30,91 & 29,29 & 28,26 & 31,17 & 29,91 \\
\hline $\begin{array}{l}\text { Deuda } \\
\text { Bancaria } \\
\text { Corto Plazo. }\end{array}$ & 35,11 & 25,50 & 20,85 & 31,06 & 28,13 & 17,55 & 15,29 & 15,02 & 14,38 & 15,56 \\
\hline $\begin{array}{l}\text { Gastos, } \\
\text { impuestos } \\
\text { otros } \\
\text { corrientes }\end{array}$ & 7,18 & 9,15 & 7,26 & 8,34 & 7,98 & 14,72 & 11,51 & 17,94 & 17,21 & 15,34 \\
\hline $\begin{array}{l}\text { Pasivo } \\
\text { corriente }\end{array}$ & 71,40 & 56,88 & 55,71 & 60,27 & 61,07 & 63,18 & 56,09 & 61,22 & 62,75 & 60,81 \\
\hline $\begin{array}{l}\text { Anticipos } \\
\text { recibidos }\end{array}$ & 0,72 & 0,20 & 0,64 & 0,71 & 0,67 & 6,42 & 13,31 & 10,19 & 4,49 & 8,60 \\
\hline $\begin{array}{l}\text { Préstamos } \\
\text { bancarios a } \\
\text { largo plazo }\end{array}$ & 15,07 & 32,82 & 28,54 & 24,85 & 25,32 & 16,61 & 16,91 & 16,08 & 18,27 & 16,97 \\
\hline $\begin{array}{l}\text { Retenciones } \\
\text { laborales }\end{array}$ & 3,68 & 5,93 & 6,18 & 5,04 & 5,20 & 4,28 & 3,62 & 3,89 & 4,46 & 4,06 \\
\hline $\begin{array}{l}\text { Cuentas } \\
\text { por pagar a } \\
\text { socios }\end{array}$ & 8,23 & 3,81 & 8,75 & 8,99 & 7,44 & 8,06 & 8,54 & 4,99 & 7,08 & 7,17 \\
\hline $\begin{array}{l}\text { Otros pasivos } \\
\text { a largo plazo }\end{array}$ & 0,89 & 0,36 & 0,18 & 0,15 & 0,40 & 1,47 & 1,52 & 3,63 & 2,96 & 2,39 \\
\hline $\begin{array}{l}\text { Pasivo no } \\
\text { corriente }\end{array}$ & 28,60 & 43,12 & 44,29 & 39,73 & 38,93 & 36,82 & 43,91 & 38,78 & 37,25 & 39,19 \\
\hline
\end{tabular}

Fuente: elaboración propia, a partir de información financiera obtenida del SIREM (Colombia) y de la aplicación de encuestas a empresas venezolanas.

Al puntualizar los subtotales de los pasivos clasificados según su vencimiento, se observan importantes similitudes; durante el periodo ambos grupos de empresas se financian mayoritariamente con pasivos corrientes o circulantes, consistentes en deuda bancaria de corto plazo, deuda comercial y pagos diferidos; cuentas que en conjunto agrupan un $61 \%$ de los pasivos, es decir, ambos parques industriales se financian en un $61 \%$ con pasivo corriente y un $39 \%$ con deudas no corriente, aun cuando internamente la distribución y jerarquización de los pasivos 
según su naturaleza varía entre ambos grupos.

En relación a los pasivos corrientes, se observa como las PYMI venezolanas financian una porción importante de capital de trabajo con deuda bancaria, esta representa un $45 \%$ del corriente y es la cuenta de mayor peso dentro de los pasivos. Contrariamente para las PYMI colombianas, el pasivo bancario de corto plazo es solo un $25 \%$ de los pasivos corrientes y es apenas la tercera fuente de financiamiento.
El detalle del análisis vertical del período deja claro además el peso de las cuentas por pagar comerciales en el caso de las PYMI de Cúcuta, siendo el pasivo más representativo con un promedio de un $29,9 \%$ del total de las deudas, es seguido con el pasivo bancario de largo plazo con apenas un $16,9 \%$, para este grupo de empresas la distribución de las fuentes de financiamiento resulta más heterogenia que en el caso venezolano y el detalle se aprecia mejor en la Figura 2.

Figura 2

\section{Conformación de las fuentes de financiamiento externas de las PYMI colombo-venezolanas (Promedio del período 2009-2012)}
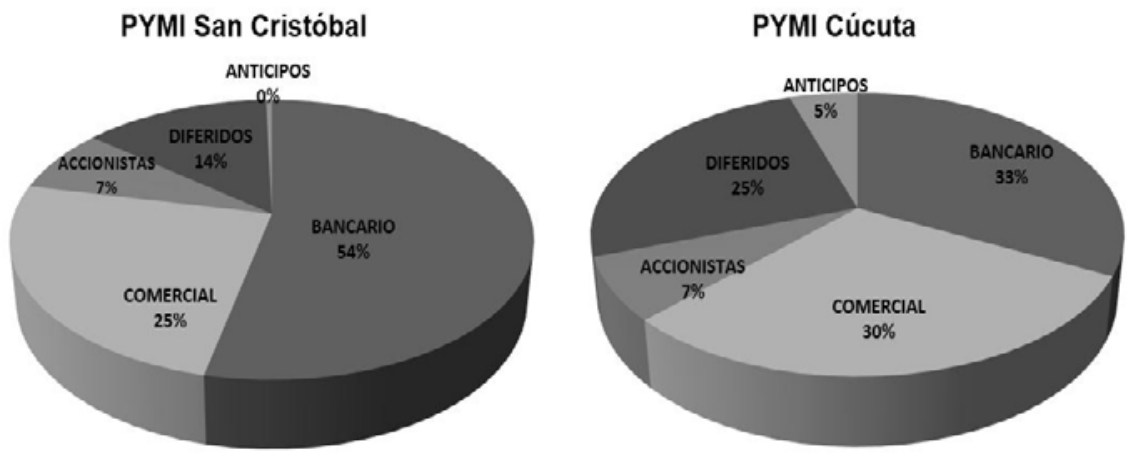

Fuente: elaboración propia, a partir de información financiera obtenida del SIREM (Colombia) y de la aplicación de encuestas a empresas venezolanas.

Durante el periodo objeto de análisis, ambas partidas del pasivo bancario (de largo y de corto plazo) tienen una participación muy importante en la conformación de las deudas del parque industrial venezolano (54\%), mientras que en las empresas colombianas representan un $33 \%$. Este hecho constituye a la banca como el principal acreedor del parque industrial de San Cristóbal y permite inferir una mayor accesibilidad a los esquemas crediticios que ofrece la banca en Venezuela, fomentado por tasas de interés reales negativas (por debajo del índice de inflación) y la existencia de un producto crediticio particular para la actividad manufacturera, regulado por una gaveta de obligatorio cumplimiento del $10 \%$ para 
la Banca $^{2}$ y que brinda plazos y tasas preferenciales.

Las deudas comerciales $y$ bancarias, agrupan para las PYMI de San Cristóbal un $79 \%$ de los pasivos y para las cucuteñas son sólo un $63 \%$. Puede hablarse de una mayor heterogeneidad en cuanto a vías de financiación para el caso colombiano aunado al uso de dos fuentes crediticias de muy bajo costo, como lo son los pagos diferidos (gastos e impuestos por pagar) y los anticipos recibidos de clientes, con un $30 \%$ de participación en la distribución de los pasivos, contra apenas un $14 \%$ en el caso venezolano. La otra porción de la estructura financiera la comprende el patrimonio, cuyo detalle se recoge en la Tabla 3.

Tabla 3

Estructura del patrimonio de las PYMI colombo-venezolanas (Período 2009-2012)

\begin{tabular}{|c|c|c|c|c|c|c|c|c|c|c|}
\hline \multirow[b]{2}{*}{ PATRIMONIO } & \multicolumn{5}{|c|}{$\begin{array}{l}\text { Estructura del patrimonio de las } \\
\text { PYMI de San Cristóbal (\%) }\end{array}$} & \multicolumn{5}{|c|}{$\begin{array}{c}\text { Estructura del patrimonio de las } \\
\text { PYMI de Cúcuta (\%) }\end{array}$} \\
\hline & 2009 & 2010 & 2011 & 2012 & Prom & 2009 & 2010 & 2011 & 2012 & Prom \\
\hline Capital social & 59,73 & 37,38 & 25,93 & 23,71 & 36,69 & 16,30 & 17,35 & 16,25 & 21,57 & 17,87 \\
\hline $\begin{array}{l}\text { Utilidades no } \\
\text { Distribuidas }\end{array}$ & 34,37 & 54,01 & 55,13 & 71,75 & 53,82 & 47,15 & 48,01 & 47,78 & 36,20 & 44,78 \\
\hline Reservas & 5,90 & 8,60 & 18,94 & 4,54 & 9,49 & 36,55 & 34,64 & 35,97 & 42,24 & 37,35 \\
\hline
\end{tabular}

Fuente: elaboración propia, a partir de información financiera obtenida del SIREM (Colombia) y de la aplicación de encuestas a empresas venezolanas.

La conformación porcentual del patrimonio en ambos grupos concentra una mayor proporción en fuentes no capitalizadas (superávit y otras reservas) aun cuando estos recursos fortalecen la estructura financiera y son fuente de reinversión y eventual incremento del capital social, podrían suprimirse por decreto de dividendos en efectivo, evento cuya posibilidad aumenta si las empresas poseen una fuerte pro-porción de activos en circulantes de rápida realización. Destaca además cierta diferencia en los niveles de capitalización, esto es, el porcentaje de capital social suscrito y pagado respecto del total patrimonio marcadamente superior en las empresas venezolanas $(37 \%$ contra solo un $18 \%$ en el caso de las PYMI colombianas).

\section{Análisis comparativo de los indicadores financieros de las PYMI colombo-venezolanas}

Ofrecida una caracterización a nivel de la estructura de activos y financiera, se describe a continuación cómo esa connotación deriva en un conjunto de razones financieras de amplia aplicación, agrupadas en los módulos de solvencia, actividad, apalancamiento y rentabilidad. En la Figura 3 se aprecia como los valores

2 Ley para la Promoción y Desarrollo de la Pequeña y Mediana Industria y Unidades de Propiedad Social, publicada en Gaceta Oficial Extraordinaria № 5.890 del 31/07/08 (Ministerio del Poder Popular para la Economía Comunal, 2008) 
de solvencia corriente, liquidez y solidez son significativamente superiores para el pequeño y mediano parque industrial cucuteño.

\section{Figura 3}

\section{Comportamiento de los indicadores de solvencia, liquidez y solidez de las PYMI colombo-venezolanas (Promedio del período 2009-2012)}
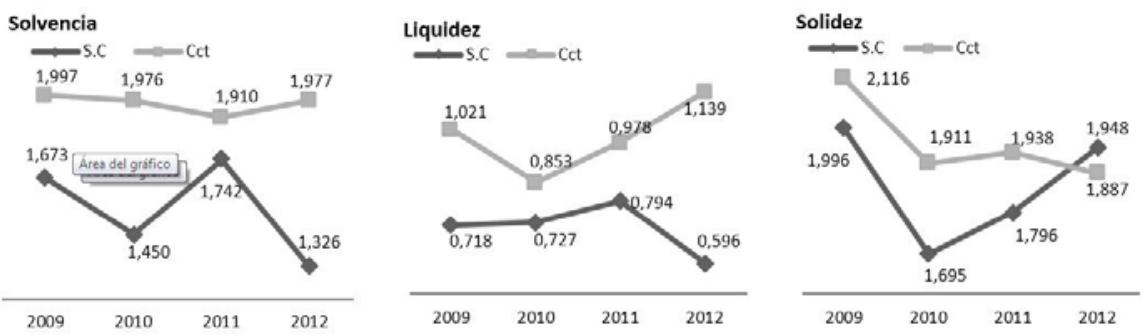

\section{S.C.: San Cristóbal; Cct: Cúcuta}

Fuente: elaboración propia, a partir de información financiera obtenida del SIREM (Colombia) y de la aplicación de encuestas a empresas venezolanas.

El primer indicador, conocido generalmente como solvencia corriente, contrasta los activos circulantes (disponible, exigible y realizable) con los pasivos de corto plazo y evidencia como ambos grupos de empresas poseen capacidad para afrontar sus compromisos de corto plazo, resulta estable y ligeramente superior para el caso de las empresas colombianas; los indicadores del parque industrial venezolano marcan una ligera tendencia a la disminución.

El indicador de liquidez, también conocido como prueba ácida o súper ácida, similar a la solvencia, con la diferencia de considerar sólo el activo disponible (efectivo y equivalentes) y exigible (cuentas por cobrar comerciales y efectivas) versus la totalidad de los pasivos corrientes; determina la capacidad que posea la organización de cubrir sus deudas de corto plazo sin necesidad de recurrir a los inventarios.
La diferencia entre el valor de solvencia y el valor de liquidez pone en evidencia la dependencia de la organización a los inventarios y a su adecuada gestión. Se denota como el parque industrial de San Cristóbal posee una mayor dependencia a los inventarios ya que en ninguno de los períodos evaluados reporta capacidad para cubrir sus deudas de corto plazo sin recurrir a los mismos, aun cuando posee en términos porcentuales un mayor nivel de efectivo como se mostró en el detalle de la estructura económica. La Figura 3 evidencia como los niveles de liquidez de las empresas colombianas reportan una tendencia creciente, mientras que las venezolanas experimentan un comportamiento contrario.

El indicador de solidez, contrasta la totalidad de los activos con los pasivos totales, muestra un comportamiento contrario a los anteriores, esto es, tendencia decreciente del lado cucuteño y estable o 
ligeramente creciente en el caso de San Cristóbal. El comportamiento opuesto entre la tendencia de los niveles de solidez versus los de liquidez y solvencia, apunta a una posible mayor inversión en activos fijos operativos en el caso de las PYMI de San Cristóbal, lo cual se ratifica al observar la evolución de los niveles porcentuales de los activos fijos de la Tabla 1, en la cual se evidenció la marcada tendencia decreciente del porcentaje de participación de los activos fijos netos en el caso de las PYMl cucuteñas.

Respecto a los índices de actividad, también conocidos como calidad y eficiencia de los activos, comprende la rotación de las cuentas por cobrar (comerciales propias del giro $\mathrm{u}$ actividad principal de la empresa), los inventarios y las cuentas por pagar (a proveedores), así como sus indicadores complementarios de días medios o "Días A Mano" (DAM), que resultan incluso más ilustrativos.

En la Tabla 4 se exponen los valores de los indicadores de actividad, en específico los días medios de realización de cuentas por cobrar comerciales (DAM CxC o plazo promedio de cobro), días medios de inventario (DAM inventarios o días medio de rotación) y de cuentas por pagar comerciales (DAM CxP o plazo promedio de pago) así como también el llamado "Ciclo Operativo" y "Ciclo de la caja", relacionados todos con el giro u actividad principal para ambos grupos de empresas a valores promedios.

\section{Tabla 4 \\ Índices de actividad de las PYMI colombo-venezolanas} (Período 2009-2012)

\begin{tabular}{|c|c|c|c|c|c|c|c|c|c|c|}
\hline \multirow[b]{2}{*}{$\begin{array}{l}\text { MÓDULO DE } \\
\text { ACTIVIDAD }\end{array}$} & \multicolumn{5}{|c|}{$\begin{array}{l}\text { Índices de actividad de las PYMI } \\
\text { de San Cristóbal }\end{array}$} & \multicolumn{5}{|c|}{$\begin{array}{l}\text { Índices de actividad de las PYMI } \\
\text { de Cúcuta }\end{array}$} \\
\hline & 2009 & 2010 & 2011 & 2012 & Prom & 2009 & 2010 & 2011 & 2012 & Prom \\
\hline $\begin{array}{l}\text { Rotación de } \\
\text { cuentas por } \\
\text { cobrar }\end{array}$ & 10,97 & 12,24 & 9,81 & 14,76 & 11,94 & 6,52 & 7,35 & 5,95 & 4,36 & 6,05 \\
\hline $\begin{array}{l}\text { DAM Cuentas } \\
\text { por Cobrar }\end{array}$ & 32,83 & 29,41 & 36,71 & 24,39 & 30,83 & 55,24 & 48,95 & 60,50 & 82,51 & 61,80 \\
\hline $\begin{array}{l}\text { Rotación de } \\
\text { Inventario }\end{array}$ & 3,07 & 4,89 & 3,28 & 4,72 & 3,99 & 3,82 & 3,27 & 3,83 & 3,85 & 3,69 \\
\hline DAM Inventario & 117,41 & 73,59 & 109,59 & 76,33 & 94,23 & 94,20 & 110,01 & 93,95 & 93,53 & 97,92 \\
\hline $\begin{array}{l}\text { Rotación Cuen- } \\
\text { tas por Pagar }\end{array}$ & 7,18 & 9,05 & 6,28 & 9,94 & 8,11 & 7,62 & 7,04 & 7,74 & 6,49 & 7,22 \\
\hline $\begin{array}{l}\text { DAM Cuentas } \\
\text { por Pagar }\end{array}$ & 50,12 & 39,78 & 57,28 & 36,22 & 45,85 & 47,24 & 51,15 & 46,53 & 55,47 & 50,10 \\
\hline Ciclo Operativo & 150,24 & 103,00 & 146,30 & 100,72 & 125,06 & 149,43 & 158,96 & 154,44 & 176,04 & 159,72 \\
\hline Ciclo de la Caja & 100,11 & 63,22 & 89,02 & 64,50 & 79,21 & 102,19 & 107,81 & 107,91 & 120,57 & 109,62 \\
\hline
\end{tabular}

Fuente: elaboración propia, a partir de información financiera obtenida del SIREM (Colombia) y de la aplicación de encuestas a empresas venezolanas. 
Se destaca en primer lugar como las PYMl de Cúcuta rotan sus cuentas por cobrar menos veces al año que sus homólogas en San Cristóbal, en otras palabras recuperan sus cuentas por cobrar prácticamente en el doble del tiempo, sugiriendo una mayor flexibilización de las políticas de crédito y cobranza en el caso de las empresas colombianas. Nuevamente esta diferenciación puede ser explicada por la diferencia en los niveles de inflación, como expone García (2001:199) las cuentas por cobrar son partidas monetarias y provocan pérdidas monetarias en épocas inflacionarias. Un ambiente inflacionario en Venezuela impone mayor rigor a nivel de las políticas de crédito.

En cuanto a la realización de los inventarios, no se aprecian diferencias significativas, los valores resultan ser mucho más estables para el parque industrial colombiano con un promedio en los últimos cuatro años de 98 días. Las empresas venezolanas presentan promedios con mayor variabilidad, reportando un máximo de 117 días y mínimos de 73 días.

El promedio en días para liquidación de las cuentas por pagar a proveedores ostenta estabilidad y valores ligeramente superiores en las PYMI cucuteñas. La comparación de plazos promedio de cobro respecto a los plazos promedio de pago devela que el parque industrial de San Cristóbal goza de financiamiento espontáneo, particularidad presentada cuando la cobranza se lleva a cabo con mayor frecuencia al pago a proveedores, en este caso ejecutan la cobranza en promedios de 31 días y sus proveedores conceden crédito en promedio de 46 días, lo cual genera cierta holgura en beneficio de manejo del efectivo, situación que no se presenta en las PYMI de Cúcuta.

El penúltimo indicador mostrado en la Tabla 4 corresponde al ciclo operativo, es decir, el tiempo promedio en que los recursos salen de caja vía compras, pasando por la reposición de las materias primas, su procesamiento, permanencia en stock, despacho como mercancía terminada, hasta su recuperación por la vía de las cobranzas, momento en que el efectivo retorna a caja. Para el pequeño y mediano parque industrial de San Cristóbal el promedio del referido indicador para los cuatro años es de 125 días, para el cucuteño de 160 días; diferencia adjudicable a los períodos de cobranza marcadamente superiores.

En el último renglón de la Tabla 4, se muestra el indicador de "ciclo de la caja". Este índice se obtiene al descontar del ciclo del efectivo el plazo promedio de financiamiento conferido por los proveedores, es un estimador de las necesidades de previsión efectivo para fines operacionales o del plazo en días que se requerirá financiar capital de trabajo. Para las PYMI de San Cristóbal es solo de 79 días, para las de Cúcuta se reporta un requerimiento de financiamiento de 125 días.

El tercer módulo a evaluar corresponde a los indicadores de endeudamiento comprendidos por el endeudamiento general y las razones de autonomía y apalancamiento cuyo detalle se muestra en la Tabla 5. 


\section{Tabla 5 \\ Índices de endeudamiento de las PYMI colombo-venezolanas (Período 2009-2012)}

\begin{tabular}{|c|c|c|c|c|c|c|c|c|c|c|}
\hline \multirow[b]{2}{*}{$\begin{array}{c}\text { Módulo de } \\
\text { endeudamiento }\end{array}$} & \multicolumn{5}{|c|}{$\begin{array}{c}\text { Índices de actividad de las PYMI } \\
\text { de San Cristóbal }\end{array}$} & \multicolumn{5}{|c|}{$\begin{array}{l}\text { Índices de actividad de las PYMI } \\
\text { de Cúcuta }\end{array}$} \\
\hline & 2009 & 2010 & 2011 & 2012 & Prom & 2009 & 2010 & 2011 & 2012 & Prom \\
\hline $\begin{array}{l}\text { Endeudamiento } \\
\text { General }(\%)\end{array}$ & 100,43 & 143,86 & 125,63 & 105,46 & 118,85 & 89,58 & 109,82 & 106,58 & 112,74 & 104,68 \\
\hline $\begin{array}{l}\text { Endeudamiento } \\
\text { de Corto Plazo } \\
(\%)\end{array}$ & 71,71 & 81,83 & 69,99 & 63,56 & 71,77 & 56,59 & 61,60 & 65,24 & 70,75 & 63,55 \\
\hline $\begin{array}{c}\text { Autonomía } \\
\text { Financiera (\%) }\end{array}$ & 49,89 & 41,01 & 44,32 & 48,67 & 45,97 & 52,75 & 47,66 & 48,41 & 47,01 & 48,96 \\
\hline $\begin{array}{c}\text { Apalancamiento } \\
(\%)\end{array}$ & 50,11 & 58,99 & 55,68 & 51,33 & 54,03 & 47,25 & 52,34 & 51,59 & 52,99 & 51,04 \\
\hline
\end{tabular}

Fuente: elaboración propia, a partir de información financiera obtenida del SIREM (Colombia) y de la aplicación de encuestas a empresas venezolanas.

En la Tabla 5 se listan el índice de endeudamiento general (contrasta el pasivo total versus el patrimonio), el índice de endeudamiento de corto plazo (cociente entre los pasivos de corto plazo y el patrimonio) y los índices complementarios de Autonomía Financiera (E) y Apalancamiento Financiero (D), también conocidos como indicadores de la estructura de capital, resultan de dividir el total patrimonio y el total pasivo respectivamente sobre el total activo, ambos son complementarios ya que su suma es igual a $100 \%$.

Destaca como el indicador de endeudamiento general para ambos grupos de empresas es superior al $100 \%$, con ello, los pasivos superan en promedio al patrimonio. Esto es una situación de endeudamiento para ambos grupos, ligeramente superior para las PYMI de San Cristóbal.

El indicador de autonomía señala la porción de la estructura de activos soportada por recursos propios de la empresa, en otras palabras la proporción de los activos en manos de los accionistas y recíprocamente el indicador de apalancamiento corresponderá a la porción de los activos soportada por los acreedores. En el caso del parque industrial cucuteño la autonomía se acerca a un $49 \%$ y el apalancamiento de $51 \%$, pudiendo considerarse bastante equilibrada. En el caso de las empresas de San Cristóbal la autonomía financiera es muy cercana a un $46 \%$ y el apalancamiento a un $54 \%$, en otras palabras en el caso de las PYMI venezolanas la estructura de activos es mayormente auspiciada por pasivos (acreedores).

La Figura 4 muestra la tendencia interanual del índice de endeudamiento general y autonomía financiera. Nótese como el índice de endeudamiento general en el caso de las PYMI de San Cristóbal reporta una tendencia decreciente $y$ consecuentemente el índice de autonomía 
Estructura económico-financiera de pequeñas y medianas empresas...

Oliveros Delgado, Juan Alberto

reporta una tendencia creciente; por el contrario en el caso de las PYMI de Cúcuta el endeudamiento reporta una tendencia creciente y la autonomía decreciente. Particularidad en la que podría ejercer influencia los resultados del negocio expuestos a través del módulo de rentabilidad a continuación en la Figura 5.

Figura 4

Comportamiento de los indicadores de endeudamiento general y de autonomía financiera de las PYMI colombo-venezolanas (Promedio del período 2009-2012)
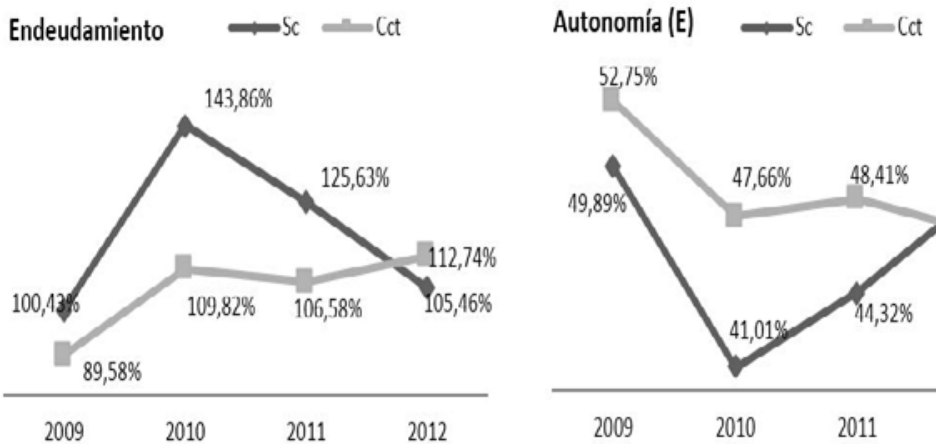

Sc: San Cristóbal; Cct: Cúcuta

Fuente: elaboración propia, a partir de información financiera obtenida del SIREM (Colombia) y de la aplicación de encuestas a empresas venezolanas.

\section{Figura 5}

\section{Comportamiento de los indicadores de rentabilidad de las PYMI colombo-venezolanas (Promedio del período 2009-2012)}
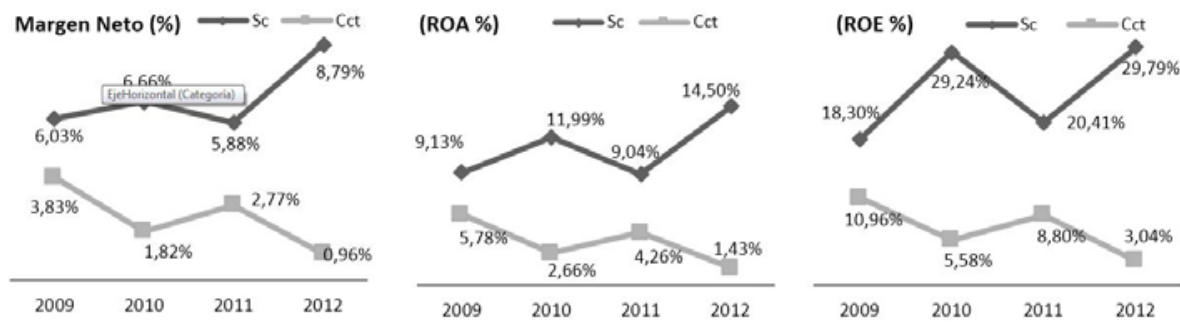

Sc: San Cristóbal; Cct: Cúcuta

Fuente: elaboración propia, a partir de información financiera obtenida del SIREM (Colombia) y de la aplicación de encuestas a empresas venezolanas. 
Se aprecia como para los tres indicadores los resultados del pequeño y mediano parque industrial de San Cristóbal son comparativamente superiores y con tendencia creciente, las diferencias significativas están influenciadas por los escenarios macroeconómicos diferentes.

Vale acotar que la marcada diferenciación de los valores porcentuales de los indicadores para los dos grupos en estudio no resultan concluyentes desde el punto de vista comparativo, pues deben ser evaluados respecto a variables propias del entorno económico de cada país, como las tasas de interés activas y pasivas, el costo de los capitales y el incremento en el índice de general de precios. No obstante las tendencias de un lado crecientes (Venezuela) y del otro decrecientes (Colombia) son dignas de apreciación y explican el por qué de las variaciones de los índices de endeudamiento y autonomía expuestos en el apartado anterior.
En el caso del parque industrial venezolano la marcada y creciente tendencia en el margen neto acompañada de una importante reinversión de beneficios en la operatividad de las empresas permite reforzar la situación patrimonial y con ello favorecer a la autonomía financiera y disminuir el endeudamiento. En las PYMI colombianas se observa una situación inversa, una baja en los resultados de los negocios ha conllevado a una menor reinversión e incrementar el financiamiento, aumentado el apalancamiento financiero y el endeudamiento general.

En la Tabla 6 se muestra nuevamente el detalle de los indicadores considerados para el módulo de rentabilidad y se hace la analogía del resultado financiero respecto a la inflación anual en cada país para determinar una posición de protección o destrucción de los capitales respecto a la inflación.

\section{Tabla 6}

\section{Índices de rentabilidad de las PYMI colombo-venezolanas e índices de inflación en Venezuela y Colombia (Período 2009-2012)}

\begin{tabular}{|c|c|c|c|c|c|c|c|c|c|c|}
\hline \multirow{2}{*}{$\begin{array}{c}\text { INDICADORES } \\
\text { Módulo de Rentabilidad }\end{array}$} & \multicolumn{5}{|c|}{$\begin{array}{c}\text { Índices de rentabilidad de las PYMI } \\
\text { de San Cristóbal e } \\
\text { indices de inflación de Venezuela }\end{array}$} & \multicolumn{5}{|c|}{$\begin{array}{l}\text { Índices de actividad de las PYMI } \\
\text { de Cúcuta e } \\
\text { índices de inflación de Colombia }\end{array}$} \\
\hline & 2009 & 2010 & 2011 & 2012 & Prom & 2009 & 2010 & 2011 & 2012 & Prom \\
\hline Resultado Operativo (\%) & 8,96 & 10,42 & 10,86 & 12,86 & 10,78 & 6,89 & 3,88 & 4,97 & 5,06 & 5,20 \\
\hline Resultado Neto (\%) & 6,03 & 6,66 & 5,88 & 8,79 & 6,84 & 3,83 & 1,82 & 2,77 & 0,96 & 2,34 \\
\hline Rentabilidad Económica (ROA \%) & 9,13 & 11,99 & 9,04 & 14,50 & 11,17 & 5,78 & 2,66 & 4,26 & 1,43 & 3,53 \\
\hline Rentabilidad Financiera (ROE\%) & 18,30 & 29,24 & 20,41 & 29,79 & 24,43 & 10,96 & 5,58 & 8,80 & 3,04 & 7,10 \\
\hline Inflación Anual Oficial (\%) & 25,10 & 27,20 & 27,60 & 20,10 & 25,00 & 2,00 & 3,17 & 3,73 & 2,44 & 2,84 \\
\hline $\begin{array}{l}\text { Generación (Destrucción) de } \\
\text { valor respecto a la Inflación (\%) }\end{array}$ & $-6,80$ & 2,04 & $-7,19$ & 9,69 & $-0,57$ & 8,96 & 2,41 & 5,07 & 0,60 & 4,26 \\
\hline
\end{tabular}

Fuente: elaboración propia, a partir de información financiera obtenida del SIREM (Colombia) y de la aplicación de encuestas a empresas venezolanas. 
Se observa como aun cuando los resultados en general resultan muy superiores en las PYMI venezolanas al compararlos con el índice de inflación anual oficial, determina una situación de destrucción de la inversión en términos monetarios (resultados del 2009, 2011 y promedio de los cuatro años), donde el escenario macroeconómico del 2013, el más desfavorable en los últimos quince años, (no considerado a efectos del presente trabajo), estima un deterioro todavía mayor.

Por el contrario en las empresas colombianas, aun cuando los resultados son comparativamente muy inferiores, la marcada tendencia a la disminución en el índice general de precios, permite pese al deterioro a nivel porcentual de los resultados del negocio, mantener las inversiones protegidas respecto a la inflación (desde el punto de vista de pérdida o ganancia por efecto inflacionario). El escenario de inflación del 2013, la más baja en los últimos quince años acentúa esta tendencia proteccionista.

\section{Conclusiones}

A la luz de las cifras mostradas en las secciones anteriores se pueden resumir importantes diferencias tanto en la estructura económica, la estructura financiera y las razones financieras de los grupos estudiados.

Vistas ambas fisonomías en cuanto a decisiones de inversión, puede aseverarse que la disposición de la estructura económica de las empresas del lado colombiano se orienta de mejor manera con el ciclo operativo, razón de ser del negocio, mientras que la jerarquización en cuanto a inversión del lado venezolano obedece más a maniobras de protección contra la inflación y devaluación.
Las diferencias se extienden también a la jerarquización en cuanto al financiamiento, el principal proveedor de recursos de la PYMI de San Cristóbal es la banca, con un contundente $54 \%$ de los pasivos totales; tasas de interés reales negativas sostenidas por varios años pueden explicar esta particularidad.

En cuanto al análisis de razones es posible concluir que el pequeño y mediano parque industrial de Norte de Santander, se muestra en términos comparativos mucho más solvente y menos dependiente de los inventarios a la hora de honrar compromisos de corto plazo.

En términos de indicadores de actividad, la recuperación de las cuentas por cobrar resulta ser mucho más eficiente para las PYMI venezolanas, la dinámica del ciclo del efectivo permite a estas últimas un ligero financiamiento espontáneo.

Al hablar de endeudamiento, las PYMI de San Cristóbal se encuentran más endeudadas respecto a sus pares de Cúcuta, la tendencia es a disminuir las brechas, dado que las primeras muestran un endeudamiento en franca disminución y las segundas en crecimiento progresivo. De mantenerse este comportamiento podría en el mediano plazo, ejercer una marcada influencia en los resultados del negocio y niveles de solvencia.

Sorpresivamente en términos de resultados la PYMI de San Cristóbal muestra valores muy superiores y con marcada tendencia creciente, las Cucuteñas inversamente, muestran resultados con tendencia a la disminución progresiva, disentir sus resultados en términos cuantitativos no es del todo adecuado desde el punto de vista comparativo, pues deben evaluados respecto a variables propias del entorno económico.

En el caso venezolano la marcada y creciente tendencia en cuanto 
a resultados y rentabilidades, resulta alentador, lo que acompañado de una importante reinversión de beneficios en la operatividad de las empresas refuerzan la situación patrimonial y favorecen la autonomía financiera. Del lado colombiano ocurre una situación inversa, una baja en los resultados, ha conllevado a una menor reinversión de fondos propios con el consecuente incremento del financiamiento externo, aumentado el apalancamiento financiero y el endeudamiento general.

Es inevitable el impacto del entorno macroeconómico en la gestión empresarial, así un panorama alentador descrito anteriormente del lado venezolano, se ve amilanado por una inflación creciente, colocando resultados tan satisfactorios en una situación de destrucción de valor; paradójicamente los resultados poco idóneos y decrecientes del lado colombiano se ven atenuados en cuanto a protección de la inversión por una estable valorización monetaria y la inflación más baja en quince años.

Las pequeñas y medianas industrias de San Cristóbal, ante un ambiente inflacionario, procuran proteger su estructura económica a través de una alta concentración en partidas no monetarias, acortan sus plazos de crédito y ciclo de efectivo y acceden a productos de la banca en aprovechamiento de un tipo de interés negativo, invirtiendo en activos fijos productivos en un afán por generar rendimientos superiores a la creciente inflación.

Las PYMI colombianas, por su parte, ante un ambiente de baja inflación, dan mayor importancia a las partidas no monetarias en la estructura económica, flexibilizan sus cobranzas y ante tipos de interés superiores a la inflación, optan por financiarse con sus proveedores y clientes.

\section{Referencias bibliográficas}

Banco Central de Venezuela (2012), Información Estadística, PIB a precios constantes. Disponible en: http://www.bcv.org.ve/excel/5_2_4.xls. Fecha de consulta: enero 2013 .

Banco Interamericano de Desarrollo -BID(2007), Observatorio de la Micro Pequeña y Mediana Empresa. Compilación estadística para 12 países de la región. Disponible en: http:// services.iadb.org/wmsfiles/products/ Publications/381255.pdf.

Congreso de la República de Colombia (2004), Ley sobre Promoción del Desarrollo de la Micro, Pequeña y Mediana Empresa Colombiana. Diario Oficial № 45.628 de 2 de agosto, Colombia.

Departamento Administrativo Nacional de Estadística -DANE- (2013), Información Estadística Índices de Precios al Consumidor. Disponible en: http://www.dane.gov.co/index.php/ indices-de-precios-y-costos/indice-deprecios-al-consumidor-ipc. Fecha de consulta: Enero 2014

García, Alberto (2001), Análisis e Interpretación de la Información Financiera Reexpresada, Editorial CECSA, México DF.

Gitman, Lawrence y Zutter, Chad (2012), Principios de Administración Financiera. Décimo segunda edición, Pearson, México DF.

Higuerey, Ángel (2007), Administración del efectivo. Mérida: Consejo de Publicaciones de la Universidad de los Andes. Venezuela.

Instituto Nacional de Estadística (2007), Directorio Industrial 2007. Publicado por CONINDUSTRIA. Febrero 2008.

Lizcano, Jesús. (2004), Rentabilidad Empresarial, propuesta practica de análisis y evaluación. Casa Editorial de la Cámara de Comercio de Madrid, España.

Martínez, Cuauhtémoc (2006), Pequeña y Mediana Empresa: Incentivos para nuevas inversiones. Ponencia presentada en el Trigésimo Sexto 


\section{Congreso Internacional de CONINDUSTRIA, Caracas-Venezuela.}

Ministerio del Poder Popular para la Economía Comunal (2008), Ley para la Promoción y Desarrollo de la Pequeña y Mediana Industria y Unidades de Propiedad Social. Gaceta Oficial Extraordinaria № 5.890 , Venezuela.

Montoya, Alexandra; Montoya, Iván y Castellanos, Oscar (2010), Situación de la competitividad de las Pyme en Colombia: elementos actuales y retos, Agronomía Colombiana, Vol. 28; $\mathrm{N}^{\circ}$ 1, Colombia, Universidad Nacional de Colombia, pp 107-117.

Ortega, Alfonso (2002). Introducción a las finanzas. Primera edición, Mc GrawHill. Ciudad de México.

Presidencia de la República (1994), Decreto de Reforma Parcial del Código Orgánico Tributario. Gaceta Oficial Extraordinaria $\mathrm{N}^{\circ} 4.727$, Venezuela.

Presidencia de la República (2008), Decreto con rango, valor y fuerza de Ley para la Promoción y Desarrollo de la Pequeña y Mediana Industria y Unidades de Propiedad Social. Gaceta Oficial N 38.999 del 21 de agosto, Venezuela.

Rivera, Jorge y Ruíz, Daniel (2011), Análisis del desempeño financiero de empresas innovadoras del Sector Alimentos y
Bebidas en Colombia, Pensamiento \& Gestión, Nㅜ 31, Colombia, Universidad del Norte, pp 109-136.

Sánchez, Inocencio (2004), Tópicos financieros, Tópico $\mathrm{N}^{\circ} 02$, Contabilidad y estados financieros. Universidad de Carabobo. Disponible en: http://www.inosanchez. com/files/mda/af/TOPICO13_EL\%20 VALOR\%20DE\%20LA\%20EMPRESA. pdf. Fecha de consulta: septiembre 2013.

Stangl, Hermann (2011), Gerencia de Valor y MYPYMES. Análisis de las principales causas de quiebra. Disponible en: http://www.stangl.com. co. Fecha de consulta: enero 2013.

Superintendencia de Sociedades (2013), Sistema de Información y Reporte Empresarial. Disponible en: http:// sirem.supersociedades.gov.co/. Fecha de consulta: Febrero 2014.

Vera-Corina, Mary (2008), Planificación financiera y acceso al financiamiento en pequeñas y medianas empresas manufactureras venezolanas. Tesis Doctoral. Facultad de Ciencias Económicas de la Universidad del Zulia.

\section{Entrevistas}

Gómez, Henry; Presidente de la Asociación de Micros, Pequeños y Medianos empresarios de Venezuela, Caracas 15 de septiembre del 2014. 